\title{
Commuting Differential Operators of Rank 3 Associated to a Curve of Genus 2
}

Dafeng $Z U O^{\dagger \ddagger}$

$\dagger$ School of Mathematical Science, University of Science and Technology of China, Hefei 230026, P.R. China

E-mail: dfzuo@ustc.edu.cn

$\ddagger W u$ Wen-Tsun Key Laboratory of Mathematics, USTC, Chinese Academy of Sciences, P.R. China

Received March 12, 2012, in final form July 12, 2012; Published online July 15, 2012

http://dx.doi.org/10.3842/SIGMA.2012.044

Abstract. In this paper, we construct some examples of commuting differential operators $L_{1}$ and $L_{2}$ with rational coefficients of rank 3 corresponding to a curve of genus 2 .

Key words: commuting differential operators; rank 3; genus 2

2010 Mathematics Subject Classification: 13N10; 14H45; 34L99; 37K20

\section{Introduction}

The study of the commutation equation

$$
\left[L_{1}, L_{2}\right]=0
$$

of two scalar differential operators

$$
L_{1}=\frac{d^{n}}{d x^{n}}+\sum_{i=0}^{n-1} f_{i}(x) \frac{d^{i}}{d x^{i}} \quad \text { and } \quad L_{2}=\frac{d^{m}}{d x^{m}}+\sum_{j=0}^{m-1} g_{j}(x) \frac{d^{j}}{d x^{j}}, \quad n<m
$$

is one of the classical problems of the theory of ordinary differential equations.

Burchnall and Chaundy in $[1,2,3]$ have shown that "each pair of commuting operators $L_{1}$ and $L_{2}$ is connected by a nontrivial polynomial algebraic relation $Q\left(L_{1}, L_{2}\right)=0$ ". The equation $Q(z, w)=0$ determines a smooth compact algebraic curve $\Xi$ of finite genus $g$. For a generic point $P \in \Xi$, there exist common eigenfunctions $\psi(x, P)$ on $\Xi$ such that $L_{1} \psi=\lambda \psi$ and $L_{2} \psi=\mu \psi$. The dimension $l$ of the space of these functions corresponding to $P \in \Xi$ is called the rank of the commuting pair $\left(L_{1}, L_{2}\right)$. For simplicity, in this paper we denote "the commuting differential operators of rank $l$ corresponding to a curve of genus $g$ " by " $(l, g)$-operators".

Burchnall and Chaundy also made significant progress in solving the commutation equation for relatively prime orders $m$ and $n$. In this case, the rank $l$ equals to 1 . The study of this case was completed by Krichever [11, 12], who also obtained explicit formulas of the function $\psi$ and the coefficients of $L_{1}$ and $L_{2}$ in terms of the Riemann $\Theta$-function. Let us remark that there are several papers related to this case, for instance [5, 6, 23, 25, 28, 29].

But for high rank case i.e. $l>1$, it is much more complicated. In [10], the problem of classifying $(l, g)$-operators was solved by reducing the computation of the coefficients to a Riemann problem. In [13, 14] I.M. Krichever and S.P. Novikov developed a method of deforming the Tyurin parameters on the moduli space of framed holomorphic bundles over algebraic curves. By using this method, in certain cases the Riemann problem can be avoided and they found 
all $(2,1)$-operators. Let us remark that J. Dixmier in [4] also discovered an example of $(2,1)$ operators with polynomial coefficients. Furthermore, P.G. Grinevich found the condition of $(2,1)$-operators with rational coefficients [7]. S.P. Novikov and P.G. Grinevich [24] clarified the spectral data related to formally self-adjoint $(2,1)$-operators. In [21] O.I. Mokhov obtained all $(3,1)$-operators. A.E. Mironov in $[17,19]$ introduced a $\sigma$-invariance to simplify the KricheverNovikov system [14] and constructed some examples of $(2,2)$-operators, $(2,4)$-operators with polynomial coefficients and also in $[18,20]$ formally self-adjoint $(2, g)$-operators and $(3, g)$ operators. Recently, an interesting paper is due to O.I. Mokhov in [22] who constructed examples of $(2 k, g)$-operators and $(3 k, g)$-operators with polynomial coefficients for arbitrary genus $g$. For more related results, please see $[8,9,13,15,16,25,26,27]$ and references therein.

The aim of this paper is to construct examples of commuting differential operators $L_{1}$ and $L_{2}$ with rational coefficients of rank 3 corresponding to a curve of genus 2 , which is different from those in [22].

\section{The commuting operators of rank 3 and genus 2}

In this section we want to construct $(3,2)$-operators. The first step is to use a $\sigma$-invariance, due to A.E. Mironov [17], to simplify the Krichever-Novikov system (2). The second step is to solve the simplified system by making a crucial hypothesis

$$
\gamma_{1}=\gamma, \quad \gamma_{2}=a \gamma, \quad \gamma_{3}=\bar{a} \gamma, \quad a=\frac{-1+\sqrt{3} \mathbf{i}}{2} .
$$

The last step is to construct the commuting differential operators $L_{1}$ and $L_{2}$.

\subsection{The general principle}

Let $\Gamma$ be a curve of genus 2 defined in $\mathbb{C}^{2}$ by the equation

$$
w^{2}=z^{6}+c_{5} z^{5}+c_{4} z^{4}+c_{3} z^{3}+c_{2} z^{2}+c_{1} z+c_{0} .
$$

On the curve $\Gamma$, there is a holomorphic involution

$$
\sigma: \Gamma \rightarrow \Gamma \quad \text { by } \quad \sigma(z, w)=(z,-w),
$$

which has six fixed ramification points. It induces an action on the space of function by $(\sigma f)(x, P)=f(x, \sigma(P))$. Let us take $q=\left(0, \sqrt{c_{0}}\right) \in \Gamma$. For a generic point $P \in \Gamma$ there exist common eigenfunctions $\psi_{j}(x, P), j=0,1,2$ with an essential singularity at $q$, of the operators $L_{1}$ and $L_{2}$. Without loss of generality, we assume that $\psi_{j}(x, P)$ are normalized by

$$
\frac{d^{i}}{d x^{i}} \psi_{j}\left(x_{0}, P\right)=\delta_{i j}
$$

where $x_{0}$ is a fixed point. Notice that on $\Gamma-\{q\}, \psi_{j}(x, P)$ are meromorphic and have six simple poles at $P_{1}, \ldots, P_{6}$ independent of $x$. Let us consider the Wronskian matrix

$$
\vec{\Psi}\left(x, P ; x_{0}\right)=\left(\begin{array}{ccc}
\psi_{0} & \psi_{1} & \psi_{2} \\
\psi_{0}^{\prime} & \psi_{1}^{\prime} & \psi_{2}^{\prime} \\
\psi_{0}^{\prime \prime} & \psi_{1}^{\prime \prime} & \psi_{2}^{\prime \prime}
\end{array}\right)
$$

of the vector-valued function $\vec{\Psi}\left(x, P ; x_{0}\right)$, and

$$
\vec{\Psi}_{x} \vec{\Psi}^{-1}=\left(\begin{array}{ccc}
0 & 1 & 0 \\
0 & 0 & 1 \\
\chi_{0} & \chi_{1} & \chi_{2}
\end{array}\right)
$$


where $\chi_{j}=\chi_{j}(x, P)$ are independent of $x_{0}$ and meromorphic functions on $\Gamma$ with six poles at $P_{1}(x), \ldots, P_{6}(x)$ coinciding with the poles of $\psi_{j}(x, P)$ at $x=x_{0}$. In a neighborhood of $q$, the functions $\chi_{j}(x, P)$ have the form

$$
\begin{aligned}
& \chi_{0}(x, P)=k+w_{0}(x)+O\left(k^{-1}\right), \quad \chi_{1}(x, P)=w_{1}(x)+O\left(k^{-1}\right), \\
& \chi_{2}(x, P)=O\left(k^{-1}\right),
\end{aligned}
$$

where $k^{-1}$ is a local parameter near $q$. The expansion of $\chi_{j}$ in a neighborhood of the pole $P_{i}(x)$ has the form

$$
\chi_{j}(x, P)=-\frac{\gamma_{i}^{\prime}(x) \alpha_{i j}(x)}{k-\gamma_{i}(x)}+d_{i j}(x)+O\left(k-\gamma_{i}(x)\right), \quad \alpha_{i 2}=1,
$$

where $k-\gamma_{i}(x)$ is a local parameter near $P_{i}(x)$ for $1 \leq i \leq 6$ and $0 \leq j \leq 2$.

Lemma 2.1 ([11]). The parameters $\gamma_{i}(x), \alpha_{i j}(x)$ and $d_{i j}(x), 1 \leq i \leq 6,0 \leq j \leq 2$ satisfy the system

$$
\begin{aligned}
& \mathrm{Eq}[i, 0]:=\alpha_{i 0}(x) \alpha_{i 1}(x)+\alpha_{i 0}(x) d_{i 2}(x)-\alpha_{i 0}^{\prime}(x)-d_{i 0}(x)=0, \\
& \mathrm{Eq}[i, 1]:=\alpha_{i 1}(x)^{2}-\alpha_{i 0}(x)+\alpha_{i 1}(x) d_{i 2}(x)-\alpha_{i 1}^{\prime}(x)-d_{i 1}(x)=0 .
\end{aligned}
$$

\subsection{Explicit forms of $\chi_{j}(x, P)$}

In this subsection, we discuss explicit forms of $\chi_{j}(x, P)$ corresponding to the curve $\Gamma$ defined by $w^{2}=1+c_{3} z^{3}+c_{4} z^{4}+z^{6}$. In order to do this, we assume that

$$
\sigma \chi_{2}(x, P)=\chi_{2}(x, P), \quad \sigma P_{s}(x)=P_{s+3}(x), \quad s=1,2,3,
$$

and

$$
\gamma_{1}=\gamma, \quad \gamma_{2}=a \gamma, \quad \gamma_{3}=\bar{a} \gamma, \quad a=\frac{-1+\sqrt{3} \mathbf{i}}{2} .
$$

Theorem 2.2. Let $\gamma$ be a solution of

$$
1+c_{3} \gamma^{3}+\gamma^{6}-6(-3)^{\frac{1}{4}} c_{4}^{\frac{1}{4}} \gamma^{\prime \frac{3}{2}}=0,
$$

then functions $\chi_{0}, \chi_{1}, \chi_{2}$ are given by the formulas

$$
\begin{aligned}
& \chi_{2}(x, P)=-\sum_{s=1}^{3} \frac{\gamma_{s}^{\prime}}{z-\gamma_{s}}-\sum_{s=1}^{3} \frac{\gamma_{s}^{\prime}}{\gamma_{s}}=\frac{3 z^{3} \gamma^{\prime}}{\gamma^{4}-z^{3} \gamma}, \\
& \chi_{1}(x, P)=\tau_{1}-\sum_{s=1}^{3} \frac{G_{s} \gamma_{s}^{\prime}}{z-\gamma_{s}}+\frac{w(z) h_{1}}{2\left(z-\gamma_{1}\right)\left(z-\gamma_{2}\right)\left(z-\gamma_{3}\right)}, \\
& \chi_{0}(x, P)=\frac{\tau_{0}}{2}+\frac{1}{2 z}-\sum_{s=1}^{3} \frac{H_{s} \gamma_{s}^{\prime}}{z-\gamma_{s}}-\frac{w(z)\left(\gamma_{1} \gamma_{2} \gamma_{3}+z h_{0}\right)}{2 z\left(z-\gamma_{1}\right)\left(z-\gamma_{2}\right)\left(z-\gamma_{3}\right)},
\end{aligned}
$$

with $G_{s}, H_{s}, \tau_{0}, \tau_{1}$ defined in (9)-(14).

Proof. By using the $\sigma$-invariance of $\chi_{2}(x, P)$, we know

$$
\gamma_{s}(x)=\gamma_{s+3}(x), \quad d_{s 2}(x)=d_{s+3,2}(x), \quad s=1,2,3 .
$$


According to the properties of $\chi_{j}(x, P)$ in (2), (3) and (5), we could assume that the functions $\chi_{j}(x, P)$ are of the form in (8) with unknown functions $G_{s}=G_{s}(x), H_{s}=H_{s}(x), \tau_{r}=\tau_{r}(x)$ and $h_{r}=h_{r}(x)$ for $s=1,2,3$ and $r=0,1$.

Substituting (6) into (8), we have

$$
\chi_{2}(x, P)=\frac{3 z^{3} \gamma^{\prime}}{\gamma^{4}-z^{3} \gamma},
$$

which yields that

$$
d_{i 2}=-\frac{2 \gamma^{\prime}}{\gamma}, \quad i=1, \ldots, 6
$$

For simplicity we use the following notations

$$
\begin{aligned}
& a_{1}=1, \quad a_{2}=a, \quad a_{3}=\bar{a}, \\
& a_{s+3}=a_{s}, \quad G_{s+3}=G_{s}, \quad H_{s+3}=H_{s}, \quad s=1,2,3 .
\end{aligned}
$$

It follows from (3) that

$$
\begin{aligned}
\alpha_{s 0}= & H_{s}+\frac{w\left(a_{s} \gamma\right) h_{0}}{6 \gamma^{2} \gamma^{\prime}}+\frac{a_{s}^{2} w\left(a_{s} \gamma\right)}{6 \gamma^{\prime}}, \quad \alpha_{s 1}=G_{s}-\frac{w\left(a_{s} \gamma\right) h_{1}}{6 \gamma^{2} \gamma^{\prime}}, \\
d_{s 0}= & \frac{\tau_{0}}{2}+\frac{a_{s}^{2}}{2 \gamma}+\frac{\gamma^{\prime} \sum_{m=1}^{2}\left(1-a_{s}^{2} a_{s+m}\right) H_{s+m}}{3 \gamma} \\
& +\frac{\left(h_{0}+2\left(a_{s} \gamma\right)^{2}\right) w\left(a_{s} \gamma\right)-\left(h_{0}+\left(a_{s} \gamma\right)^{2}\right) a_{s} \gamma w^{\prime}\left(a_{s} \gamma\right)}{6 \gamma^{3}}, \\
d_{s 1}= & \tau_{1}-\frac{G_{s+1} \gamma^{\prime}}{\left(a_{s}-a_{s+1}\right) \gamma}-\frac{G_{s+2} \gamma^{\prime}}{\left(a_{s}-a_{s+2}\right) \gamma}+\frac{\left(a_{s} \gamma w^{\prime}\left(a_{s} \gamma\right)-w\left(a_{s} \gamma\right)\right) h_{1}}{6 \gamma^{3}}, \\
\alpha_{s+3, r} & =\sigma \alpha_{s r}, \quad d_{s+3,1}=\sigma d_{s 1}, \quad r=0,1, \quad s=1,2,3 .
\end{aligned}
$$

By substituting $\alpha_{i j}$ and $d_{i j}$ into (4), we get twelve equations

$$
\mathrm{Eq}[i, 0]=0, \quad \mathrm{Eq}[i, 1]=0, \quad i=1, \ldots, 6 .
$$

We now try to solve these equations. Firstly, it follows from

$$
\mathrm{Eq}[s+3,1]-\mathrm{Eq}[s, 1]=0, \quad s=1,2,3
$$

that

$$
G_{s}=\frac{h_{1}^{\prime}-h_{0}-\left(a_{s} \gamma\right)^{2}}{2 h_{1}}+\frac{\gamma^{\prime}}{2 \gamma}-\frac{\gamma^{\prime \prime}}{2 \gamma^{\prime}}, \quad s=1,2,3 .
$$

By using (9) and $\mathrm{Eq}[s+3,0]-\mathrm{Eq}[s, 0]=0$, we get

$$
\begin{aligned}
H_{s}= & \frac{\left(h_{0}+\left(a_{s} \gamma\right)^{2}\right) h_{1}^{\prime}-2 h_{0}^{\prime}-7 a_{s}^{2} \gamma \gamma^{\prime}}{2 h_{1}}-\frac{\left(h_{0}+\left(a_{s} \gamma\right)^{2}\right)^{2}}{2 h_{1}^{2}} \\
& -\frac{h_{0} \gamma^{\prime}}{2 h_{1} \gamma}+\frac{\left(h_{0}+\left(a_{s} \gamma\right)^{2}\right) \gamma^{\prime \prime}}{2 h_{1} \gamma^{\prime}}, \quad s=1,2,3 .
\end{aligned}
$$

Furthermore, by solving

$$
\operatorname{Eq}[s+3,1]+\operatorname{Eq}[s, 1]=0, \quad \operatorname{Eq}[s+3,0]+\operatorname{Eq}[s, 0]=0,
$$


we have

$$
\begin{aligned}
\operatorname{Neq}[s, 1]:= & -\tau_{1}+\frac{h_{0}^{2}+6 h_{0}\left(a_{s} \gamma\right)^{2}+6 a_{s} \gamma^{4}-6 h_{0} h_{1}^{\prime}-6\left(a_{s} \gamma\right)^{2} h_{1}^{\prime}+3 h_{1}^{\prime 2}}{4 h_{1}^{2}} \\
& +\frac{3 h_{0}^{\prime}-h_{1}^{\prime \prime}+9 a_{s}^{2} \gamma \gamma^{\prime}}{2 h_{1}}+\frac{3 h_{0} \gamma^{\prime}-2 h_{1}^{\prime} \gamma^{\prime}}{2 h_{1} \gamma}+\frac{\gamma^{\prime \prime}}{2 \gamma}+\frac{\gamma^{\prime \prime \prime}}{2 \gamma^{\prime}} \\
& -\frac{3 \gamma^{\prime 2}}{4 \gamma^{2}}-\frac{\gamma^{\prime \prime 2}}{4 \gamma^{\prime 2}}-\frac{h_{1} \gamma^{\prime \prime}}{2 h_{1} \gamma^{\prime}}+\frac{h_{1}^{2} w\left(a_{s} \gamma\right)}{36 \gamma^{4} \gamma^{\prime 2}}=0, \quad s=1,2,3,
\end{aligned}
$$

and

$$
\begin{aligned}
\text { Neq }[s, 0]:= & -\tau_{0}-\frac{a_{s}^{2}}{\gamma}+\frac{4 h_{0}^{\prime \prime}+16 a_{s}^{2} \gamma \gamma^{\prime}+21\left(a_{s} \gamma\right)^{2}}{2 h_{1}} \\
& +\frac{\left(3 h_{0}^{\prime}+9 a_{s} \gamma \gamma^{\prime}-h_{1}^{\prime \prime}\right)\left(h_{0}+\left(a_{s} \gamma\right)^{2}\right)-4 h_{0}^{\prime} h_{1}^{\prime}-13 a_{s}^{2} \gamma \gamma^{\prime} h_{1}^{\prime}}{h_{1}^{2}} \\
& +\frac{\left(h_{0}+\left(a_{s} \gamma\right)^{2}\right)^{3}-6 h_{1}^{\prime}\left(h_{0}+\left(a_{s} \gamma\right)^{2}\right)^{2}+5 h_{1}^{\prime 2}\left(h_{0}+\left(a_{s} \gamma\right)^{2}\right)}{2 h_{1}^{3}} \\
& +\frac{6 h_{0}^{\prime} \gamma^{\prime}-h_{0} \gamma^{\prime \prime}}{h_{1} \gamma}+\frac{\left(3 h_{0}^{2}-4 h_{0} h_{1}^{\prime}\right) \gamma^{\prime}}{h_{1}^{2} \gamma}-\frac{\left(h_{0}+\left(a_{s} \gamma\right)^{2}\right) \gamma^{\prime \prime \prime}}{h_{1} \gamma^{\prime}} \\
& +\frac{\left(h_{0}+\left(a_{s} \gamma\right)^{2}\right) h_{1}^{\prime} \gamma^{\prime \prime}}{h_{1}^{2} \gamma^{\prime}}+\frac{\left(h_{0}+\left(a_{s} \gamma\right)^{2}\right) \gamma^{\prime \prime 2}}{2 h_{1} \gamma^{\prime 2}}+\frac{3 h_{0} \gamma^{\prime 2}}{2 h_{1} \gamma^{2}} \\
& -\frac{\left(h_{0}+\left(a_{s} \gamma\right)^{2}\right) h_{1} w\left(a_{s} \gamma\right)}{18 \gamma^{4} \gamma^{\prime 2}}, \quad s=1,2,3 .
\end{aligned}
$$

Let us remark that we have reduced twelve equations to six equations

$$
\mathrm{Neq}[s, 0]=0, \quad \mathrm{Neq}[s, 1]=0, \quad s=1,2,3,
$$

with four unknown functions $\tau_{1}, \tau_{0}, h_{1}$ and $h_{0}$.

Let us take

$$
h_{1}=i(-3)^{\frac{3}{4}} c_{4}^{-\frac{1}{4}} \gamma \sqrt{\gamma^{\prime}}, \quad h_{0}=\frac{i(-3)^{\frac{3}{4}}\left(\gamma \gamma^{\prime \prime}-4 \gamma^{\prime 2}\right)}{2 c_{4}^{\frac{1}{4}} \sqrt{\gamma^{\prime}}} .
$$

From Neq[1, 1$]=0$, we get

$$
\tau_{1}=\frac{4 \gamma^{\prime 2}-9 \gamma \gamma^{\prime \prime}}{2 \gamma^{2}}+\frac{4 \gamma^{\prime} \gamma^{\prime \prime \prime}-3 \gamma^{\prime \prime 2}}{4 \gamma^{\prime 2}}+\frac{i\left(\gamma^{3}-1\right)^{2}}{4 \sqrt{3 c_{4}} \gamma^{2} \gamma^{\prime}} .
$$

By using (13), we conclude that Neq[2,1] $=0$ and Neq[3,1] $=0$ always hold true.

From the equation Neq[1,0] $=0$, we obtain

$$
\begin{aligned}
\tau_{0}= & \frac{i\left(\gamma^{3}-1\right)^{2}}{\sqrt{3 c_{4}} \gamma^{3}}-\frac{1}{\gamma}-\frac{i\left(\gamma^{3}-1\right)^{2} \gamma^{\prime \prime}}{4 \sqrt{3 c_{4}} \gamma^{2} \gamma^{\prime 2}}-\frac{2 i(-3)^{\frac{3}{4}} c_{4}^{\frac{3}{4}} \gamma^{3}}{27 \gamma^{\prime \frac{3}{2}}}-\frac{i(-3)^{\frac{3}{4}}\left(\gamma^{3}-1\right)^{2}}{18 c_{4}^{\frac{1}{4}} \gamma \gamma^{\prime \frac{3}{2}}} \\
& -\frac{3 \gamma^{\prime \prime \prime}}{\gamma}+\frac{10 \gamma^{\prime} \gamma^{\prime \prime}}{\gamma^{2}}-\frac{4 \gamma^{\prime 3}}{\gamma^{3}}+\frac{\gamma^{(4)}}{\gamma^{\prime}}-\frac{5 \gamma^{\prime \prime} \gamma^{\prime \prime \prime}}{2 \gamma^{\prime 2}}+\frac{3 \gamma^{\prime \prime 3}}{2 \gamma^{\prime 3}}-\frac{3 \gamma^{\prime \prime 2}}{\gamma \gamma^{\prime}} .
\end{aligned}
$$

By using (14), both Neq[2,0] $=0$ and $N e q[3,0]=0$ reduce to the same equation

$$
1+c_{3} \gamma^{3}+\gamma^{6}-6(-3)^{\frac{1}{4}} c_{4}^{\frac{1}{4}} \gamma^{\prime \frac{3}{2}}=0,
$$

which is exactly the equation (7). Thus we complete the proof of the theorem. 
Generally, solutions of (7) are not useful for us to construct $(3,2)$-operators with "good" coefficients. But when we choose $c_{3}=2$ or -2 , there are rational solutions. In what follows let us suppose

$$
c_{3}=-2, \quad c_{4}=-\frac{\epsilon^{4}}{3888}, \quad \epsilon<0 .
$$

The equation (7) is rewritten as

$$
1-2 \gamma^{3}+\gamma^{6}+\epsilon \gamma^{\prime \frac{3}{2}}=0
$$

It is easy to check that when $\left(x+s_{0}\right)^{3}+\epsilon^{2}>0$,

$$
\gamma=\frac{x+s_{0}}{\left(\left(x+s_{0}\right)^{3}+\epsilon^{2}\right)^{\frac{1}{3}}}, \quad s_{0} \in \mathbb{C}
$$

is a solution of (15). Without loss of generality, we set $s_{0}=0$. In this case we would like to write $\gamma=\gamma(x ; \epsilon)$. As a corollary of Theorem 2.2, we have

Corollary 2.3. Let $\gamma(x ; \epsilon)=\frac{x}{\left(x^{3}+\epsilon^{2}\right)^{\frac{1}{3}}}$ be a solution of (15). Then we have

$$
\begin{aligned}
\chi_{0}(x, P)= & \frac{1}{2 z}-\frac{x^{3}\left(\epsilon^{2}+x^{3}\right)}{5832}+\frac{10\left(z^{3}-1\right)}{\kappa}+\frac{\epsilon^{2} x^{3} z}{216 \kappa} \\
& -\frac{108 w(z)+\epsilon^{2} z^{2}}{6 \kappa}-\frac{x^{3} w(z)}{2 \kappa z}+\frac{16 \epsilon^{2} z^{3}}{\kappa x^{3}}, \\
\chi_{1}(x, P)= & \frac{132 \epsilon^{2} z^{3}-x^{3}\left[204-204 z^{3}+108 w(z)+\epsilon^{2} z^{2}\right]}{12 x^{2} \kappa}, \quad \chi_{2}(x, P)=-\frac{3 \epsilon^{2} z^{3}}{x \kappa},
\end{aligned}
$$

where $\kappa=\left(\epsilon^{2}+x^{3}\right) z^{3}-x^{3}$ and $w(z)=\sqrt{1-2 z^{3}-\frac{\epsilon^{2}}{3888} z^{4}+z^{6}}$.

By using (16), let us expand $\chi_{j}(x, P)$ in a neighborhood of $z=0$

$$
\begin{aligned}
& \chi_{0}(x, P)=\frac{1}{z}+\zeta_{1}-\frac{\epsilon^{2}}{216} z+\frac{2 \epsilon^{2}}{3 x^{2}} z^{2}+O\left(z^{3}\right), \\
& \chi_{1}(x, P)=\zeta_{2}+\frac{\epsilon^{2}}{12 x^{2}} z^{2}+O\left(z^{3}\right), \quad \chi_{2}(x, P)=\frac{3 \epsilon^{2}}{x^{4}}+O\left(z^{4}\right),
\end{aligned}
$$

where

$$
\zeta_{1}=\frac{28}{x^{2}}-\frac{\epsilon^{2} x^{3}+x^{6}}{5832} \quad \text { and } \quad \zeta_{2}=\frac{26}{x^{2}} .
$$

\subsection{Commuting differential operators of rank 3}

Let $\Gamma$ be a smooth curve of genus 2 defined by the equation

$$
w^{2}=1-2 z^{3}-\frac{\epsilon^{4}}{3888} z^{4}+z^{6}
$$

on the $(z, w)$-plane.

Theorem 2.4. The operator $L_{1}$ corresponding to the meromorphic function

$$
\lambda=\frac{1+w(z)}{2 z^{3}}-\frac{1}{2}
$$


on $\Gamma$ with the unique pole at $q=(0,1)$ and $L_{1} \psi=\lambda \psi$ has the form

$$
L_{1}=\frac{d^{9}}{d x^{9}}+\sum_{n=0}^{7} f_{n} \frac{d^{n}}{d x^{n}}
$$

where

$$
\begin{aligned}
f_{0}= & \frac{152}{243}-\frac{58240}{x^{9}}-\frac{55 \epsilon^{2}}{243 x^{3}}-\frac{37 \epsilon^{4} x^{3}}{11337408}+\frac{115 \epsilon^{2} x^{6}}{11337408}+\frac{37 x^{9}}{1417176} \\
& +\frac{\epsilon^{6} x^{9}}{198359290368}+\frac{\epsilon^{4} x^{12}}{66119763456}+\frac{\epsilon^{2} x^{15}}{66119763456}+\frac{x^{18}}{198359290368}, \\
f_{1}= & \frac{58240}{x^{8}}+\frac{55 \epsilon^{2}}{243 x^{2}}-\frac{152 x}{243}+\frac{5 \epsilon^{4} x^{4}}{5668704}+\frac{2 \epsilon^{2} x^{7}}{177147}+\frac{17 x^{10}}{1417176}, \\
f_{2}= & -\frac{43200}{x^{7}}+\frac{26 \epsilon^{2}}{243 x}-\frac{73 x^{2}}{243}+\frac{\epsilon^{4} x^{5}}{1259712}+\frac{\epsilon^{2} x^{8}}{419904}+\frac{x^{11}}{629856}, \\
f_{3}= & -\frac{143 \epsilon^{2}}{1944}+\frac{19120}{x^{6}}+\frac{79 x^{3}}{486}+\frac{\epsilon^{4} x^{6}}{11337408}+\frac{\epsilon^{2} x^{9}}{5668704}+\frac{x^{12}}{11337408}, \\
f_{4}= & -\frac{4800}{x^{5}}-\frac{2 \epsilon^{2} x}{243}+\frac{16 x^{4}}{243}, \quad f_{5}=-\frac{24}{x^{4}}+\frac{\epsilon^{2} x^{2}}{216}+\frac{x^{5}}{108}, \\
f_{6}= & \frac{384}{x^{3}}+\frac{\epsilon^{2} x^{3}}{1944}+\frac{x^{6}}{1944}, \quad f_{7}=-\frac{78}{x^{2}} .
\end{aligned}
$$

Proof. By using (1), we have

$$
\psi_{j}^{\prime \prime \prime}(x, P)=\chi_{2}(x, P) \psi_{j}^{\prime \prime}(x, P)+\chi_{1}(x, P) \psi_{j}^{\prime}(x, P)+\chi_{0}(x, P) \psi_{j}(x, P) .
$$

It follows from (21) that the equation $L_{1} \psi_{j}=\lambda(z) \psi_{j}$ can be rewritten as

$$
Q_{0}(x, z) \psi_{j}(x, P)+Q_{1}(x, z) \psi_{j}^{\prime}(x, P)+Q_{2}(x, z) \psi_{j}^{\prime \prime}(x, P)=\lambda(z) \psi_{j} .
$$

According to the independence of $\chi_{0}(x, P), \chi_{1}(x, P)$ and $\chi_{2}(x, P)$ at $x=x_{0}$, we conclude that the system (22) is equivalent to three equations

$$
Q_{0}(x, z)=\lambda(z), \quad Q_{1}(x, z)=0, \quad Q_{2}(x, z)=0 .
$$

By expanding $Q_{j}(x, z)$ at $z=0$, we have

$$
0=Q_{j}(x, z)-\delta_{j}^{0} \lambda(z)=Q_{j,-2} \frac{1}{z^{2}}+Q_{j,-1} \frac{1}{z}+Q_{j 0}+O(z) .
$$

Then by solving $Q_{j,-s}=0$ for $s, j=0,1,2$, we get the coefficients of $L_{1}$ given by

$$
\begin{aligned}
f_{0}= & -1-\zeta_{1}^{3}-\frac{4 \epsilon^{2}+3}{2 x^{3}}-\zeta_{2} \zeta_{1}^{\prime} \zeta_{2}^{\prime}--\zeta_{2}^{2} \zeta_{1}^{\prime \prime}+6 \zeta_{1}^{\prime} \zeta_{1}^{\prime \prime}+3 \zeta_{1}^{\prime \prime} \zeta_{2}^{\prime \prime}+3 \zeta_{2}^{\prime} \zeta_{1}^{\prime \prime \prime} \\
& +\zeta_{1}\left(-\frac{\epsilon^{2}}{72}-3 \zeta_{2} \zeta_{1}^{\prime}+3 \zeta_{1}^{\prime \prime \prime}\right)+\zeta_{1}^{\prime} \zeta_{2}^{\prime \prime \prime}+2 \zeta_{2} \zeta_{1}^{(4)}-\zeta_{1}^{(6)}, \\
f_{1}= & -\frac{1}{4 x^{2}}+6 \zeta_{1}^{\prime 2}+9 \zeta_{1} \zeta_{1}^{\prime \prime}+12 \zeta_{2}^{\prime} \zeta_{1}^{\prime \prime}+9 \zeta_{1}^{\prime} \zeta_{2}^{\prime \prime}+3 \zeta_{2}^{\prime \prime 2}-\zeta_{2}^{2}\left(3 \zeta_{1}^{\prime}+\zeta_{2}^{\prime \prime}\right)+3 \zeta_{1} \zeta_{2}^{\prime \prime \prime} \\
& +4 \zeta_{2}^{\prime} \zeta_{2}^{\prime \prime \prime}+\zeta_{2}\left(-\frac{\epsilon^{2}}{72}-3 \zeta_{1}^{2}-3 \zeta_{1} \zeta_{2}^{\prime}-\zeta_{2}^{\prime 2}+9 \zeta_{1}^{\prime \prime \prime}+2 \zeta_{2}^{(4)}\right)-6 \zeta_{1}^{(5)}-\zeta_{2}^{(6)}, \\
f_{2}= & 3\left[-\zeta_{2}^{2} \zeta_{2}^{\prime}+5 \zeta_{1}^{\prime} \zeta_{2}^{\prime}+5 \zeta_{2}^{\prime} \zeta_{2}^{\prime \prime}-\zeta_{1} \zeta_{2}^{2}+3 \zeta_{1}\left(\zeta_{1}^{\prime}+\zeta_{2}^{\prime \prime}\right)+\zeta_{2}\left(5 \zeta_{1}^{\prime \prime}+3 \zeta_{2}^{\prime \prime \prime}\right)-5 \zeta_{1}^{(4)}-2 \zeta_{2}^{(5)}\right], \\
f_{3}= & \frac{\epsilon^{2}}{72}+3 \zeta_{1}^{2}-\zeta_{2}^{3}+9 \zeta_{1} \zeta_{2}^{\prime}+9 \zeta_{2}^{\prime 2}+3 \zeta_{2}\left(4 \zeta_{1}^{\prime}+5 \zeta_{2}^{\prime \prime}\right)-21 \zeta_{1}^{\prime \prime \prime}-15 \zeta_{2}^{(4)},
\end{aligned}
$$




$$
\begin{aligned}
& f_{4}=15 \zeta_{2} \zeta_{2}^{\prime}-\zeta_{2}\left(2\left(-3 \zeta_{1}-9 \zeta_{2}^{\prime}\right)+21 \zeta_{2}^{\prime}\right)-18 \zeta_{1}^{\prime \prime}-21 \zeta_{2}^{\prime \prime \prime}, \\
& f_{5}=3 \zeta_{2}^{2}-9 \zeta_{1}^{\prime}-18 \zeta_{2}^{\prime \prime}, \quad f_{6}=-3 \zeta_{1}-9 \zeta_{2}^{\prime}, \quad f_{7}=-3 \zeta_{2} .
\end{aligned}
$$

By substituting $\zeta_{1}$ and $\zeta_{2}$ in (17) into the above formula, we obtain explicit expressions of $f_{j}$ in $(20)$.

Next we want to look for a $12^{\text {th }}$-order differential operator

$$
L_{2}=\frac{d^{12}}{d x^{12}}+\sum_{m=0}^{10} g_{m} \frac{d^{m}}{d x^{m}}
$$

such that $\left[L_{1}, L_{2}\right]=0$. Let us sketch out our ideas and omit tedious computations. The commutation equation $\left[L_{1}, L_{2}\right]=0$ is written as

$$
0=\left[\frac{d^{9}}{d x^{9}}+\sum_{n=0}^{7} f_{n} \frac{d^{n}}{d x^{n}}, \frac{d^{12}}{d x^{12}}+\sum_{m=0}^{10} g_{m} \frac{d^{m}}{d x^{m}}\right]=\sum_{k=0}^{18} W_{k}(f, g) \frac{d^{k}}{d x^{k}},
$$

which yields that

$$
W_{k}(f, g)=0, \quad k=0, \ldots, 18 .
$$

By using eleven equations $W_{k}(f, g)=0, k=8, \ldots, 18$, we could obtain explicit forms of $g_{m}=h_{m}\left(x ; \rho_{0}, \ldots, \rho_{10-m}\right)+\rho_{11-m}$ with integral constants $\rho_{11-m}$. The last eight equations will determine some integral constants. For simplicity, we take all arbitrary parameters to be zero, and then obtain all coefficients $g_{j}$ as follows

$$
\begin{aligned}
& g_{0}=\frac{45660160}{x^{12}}-\frac{4928 \epsilon^{2}}{729 x^{6}}-\frac{20048}{729 x^{3}}-\frac{605 \epsilon^{2} x^{3}}{708588}+\frac{4553 x^{6}}{708588}+\frac{79 \epsilon^{6} x^{6}}{99179645184} \\
& +\frac{269 \epsilon^{4} x^{9}}{16529940864}+\frac{683 \epsilon^{2} x^{12}}{16529940864}+\frac{\epsilon^{8} x^{12}}{1156831381426176}+\frac{661 x^{15}}{24794911296} \\
& +\frac{\epsilon^{6} x^{15}}{289207845356544}+\frac{\epsilon^{4} x^{18}}{192805230237696}+\frac{\epsilon^{2} x^{21}}{289207845356544}+\frac{x^{24}}{1156831381426176}, \\
& g_{1}=-\frac{45660160}{x^{11}}+\frac{4928 \epsilon^{2}}{729 x^{5}}+\frac{20048}{729 x^{2}}-\frac{203 \epsilon^{4} x}{2834352}+\frac{1691 \epsilon^{2} x^{4}}{2834352}+\frac{7111 x^{7}}{708588} \\
& +\frac{55 \epsilon^{6} x^{7}}{49589822592}+\frac{127 \epsilon^{4} x^{10}}{16529940864}+\frac{217 \epsilon^{2} x^{13}}{16529940864}+\frac{325 x^{16}}{49589822592}, \\
& g_{2}=\frac{27758080}{x^{10}}-\frac{182 \epsilon^{2}}{27 x^{4}}+\frac{296}{9 x}-\frac{413 \epsilon^{4} x^{2}}{5668704}+\frac{4339 \epsilon^{2} x^{5}}{2834352}+\frac{6595 x^{8}}{1417176} \\
& +\frac{\epsilon^{6} x^{8}}{3673320192}+\frac{\epsilon^{4} x^{11}}{918330048}+\frac{5 \epsilon^{2} x^{14}}{3673320192}+\frac{x^{17}}{1836660096}, \\
& g_{3}=-\frac{5992}{729}-\frac{11567360}{x^{9}}+\frac{1028 \epsilon^{2}}{729 x^{3}}+\frac{25 \epsilon^{4} x^{3}}{1417176}+\frac{457 \epsilon^{2} x^{6}}{708588}+\frac{1393 x^{9}}{1417176} \\
& +\frac{\epsilon^{6} x^{9}}{49589822592}+\frac{\epsilon^{4} x^{12}}{16529940864}+\frac{\epsilon^{2} x^{15}}{16529940864}+\frac{x^{18}}{49589822592}, \\
& g_{4}=\frac{3395840}{x^{8}}+\frac{271 \epsilon^{2}}{243 x^{2}}-\frac{2834 x}{243}+\frac{193 \epsilon^{4} x^{4}}{11337408}+\frac{317 \epsilon^{2} x^{7}}{2834352}+\frac{307 x^{10}}{2834352} \text {, } \\
& g_{5}=-\frac{693504}{x^{7}}-\frac{13 \epsilon^{2}}{243 x}+\frac{221 x^{2}}{243}+\frac{\epsilon^{4} x^{5}}{314928}+\frac{\epsilon^{2} x^{8}}{104976}+\frac{x^{11}}{157464}, \\
& g_{6}=-\frac{167 \epsilon^{2}}{972}+\frac{86464}{x^{6}}+\frac{316 x^{3}}{243}+\frac{\epsilon^{4} x^{6}}{5668704}+\frac{\epsilon^{2} x^{9}}{2834352}+\frac{x^{12}}{5668704},
\end{aligned}
$$




$$
\begin{aligned}
g_{7} & =-\frac{672}{x^{5}}+\frac{\epsilon^{2} x}{486}+\frac{109 x^{4}}{486}, & g_{8} & =-\frac{2856}{x^{4}}+\frac{\epsilon^{2} x^{2}}{108}+\frac{x^{5}}{54}, \\
g_{9} & =\frac{824}{x^{3}}+\frac{\epsilon^{2} x^{3}}{1458}+\frac{x^{6}}{1458}, & g_{10} & =-\frac{104}{x^{2}} .
\end{aligned}
$$

Remark 2.5. By analogy with the process of getting $f_{j}$ in (20), we could obtain the above $g_{j}$ in (25) by choosing another meromorphic function with a unique pole of order 4 at $z=0$ on $\Gamma$

$$
\mu(z)=\frac{1+w(z)}{2 z^{4}}-\frac{1}{2 z}
$$

Remark 2.6. One could find another operator $L_{3}$ of order 15 from $\left[L_{1}, L_{3}\right]=0$. Furthermore as in [17], the commutative ring of differential operators generated by $L_{1}, L_{2}$ and $L_{3}$ is isomorphic to the ring of meromorphic functions on $\Gamma$ with the pole at $q=(0,1)$.

\subsection{The corresponding Burchnall-Chaundy curve}

According to the Burchnall-Chaundy's correspondence in $[1,2,3]$, for each pair of commuting operators $L_{1}$ and $L_{2}$ there is a Burchnall-Chaundy curve defined by a minimal nontrivial polynomial $Q(z, w)=0$ such that $Q\left(L_{1}, L_{2}\right)=0$ (or $Q\left(L_{2}, L_{1}\right)=0$ ). Obviously, the above curve $\Gamma$ defined by (18) is not the Burchnall-Chaundy curve for $L_{1}$ and $L_{2}$ given in (19) and (24). Actually the corresponding Burchnall-Chaundy curve $\tilde{\Gamma}$ is given by

$$
w^{3}-\frac{\epsilon^{4}}{15552} w^{2}=z^{4}+z^{3}
$$

that is to say,

$$
L_{2}^{3}-\frac{\epsilon^{4}}{15552} L_{2}^{2}=L_{1}^{4}+L_{1}^{3}
$$

The curve $\tilde{\Gamma}$ has a cuspidal singularity at $(0,0)$. The operators $L_{1}$ and $L_{2}$ correspond to those meromorphic functions on $\Gamma$

$$
\lambda=\frac{1+w(z)}{2 z^{3}}-\frac{1}{2}, \quad \mu=\frac{1+w(z)}{2 z^{4}}-\frac{1}{2 z}
$$

defining a birational equivalence

$$
\pi: \Gamma \rightarrow \tilde{\Gamma}, \quad \pi(z, w)=(\lambda, \mu) .
$$

The inverse image of the cuspidal point is the point $\sigma(q)$, where $q=(0,1) \in \Gamma$. In order to make $\pi$ to be a morphism, we must complement $\tilde{\Gamma}$ at infinity by a cuspidal point of the type $(3,4)$, then its inverse image is the point $q$.

\section{Concluding remarks}

In summary by using a $\sigma$-invariance to simplify the Krichever-Novikov system, we have constructed a pair of commuting differential operators $L_{1}$ in (19) and $L_{2}$ in (23) of rank 3 with rational coefficients corresponding to the singular curve $\tilde{\Gamma}$, which is birationally equivalent to the smooth curve $\Gamma$ of genus 2 .

Let us remark that all of coefficients of $L_{1}$ and $L_{2}$ are polynomials with respect to the parameter $\epsilon$. So if we take

$$
\mathcal{L}_{1}=\lim _{\epsilon \rightarrow 0} L_{1}, \quad \mathcal{L}_{2}=\lim _{\epsilon \rightarrow 0} L_{2},
$$


then

$$
\left[\mathcal{L}_{1}, \mathcal{L}_{2}\right]=0, \quad \mathcal{L}_{2}^{3}=\mathcal{L}_{1}^{4}+\mathcal{L}_{1}^{3}
$$

More precisely, we have

$$
\mathcal{L}_{1}=\mathcal{L}^{3}-1, \quad \mathcal{L}_{2}=\mathcal{L}^{4}-\mathcal{L},
$$

where

$$
\mathcal{L}=\frac{d^{3}}{d x^{3}}-\frac{26}{x^{2}} \frac{d}{d x}-\frac{28}{x^{3}}+\frac{x^{6}}{5832} .
$$

So, when $\epsilon=0$ this is a trivial example.

How about the case $\epsilon \neq 0$ ? Let us comment that in this case, by a direct verification there is not such kind of $\mathcal{L}$ of order 3 commuting with $L_{1}$ and $L_{2}$. Furthermore, according to the result in [29], any rank one operator with rational coefficients whose second highest coefficient is zero has the property that the limit as $x$ goes to $\infty$ of the coefficients is zero. So, for example, the absence of a $\frac{d^{11}}{d x^{11}}$ term in $L_{2}$ and the $x^{6}$ in the coefficient of its $\frac{d^{9}}{d x^{9}}$ term which means that $L_{2}$ is not a rank 1 operator.

\section{Acknowledgments}

The author is grateful to Andrey E. Mironov for bringing the attention to this project and helpful discussions. The author also thanks referees' suggestions and Alex Kasman for pointing some errors in the first version of this paper, Qing Chen and Youjin Zhang for their constant supports. This work is supported by "PCSIRT" and the Fundamental Research Funds for the Central Universities (WK0010000024) and NSFC (No. 10971209) and SRF for ROCS, SEM.

\section{References}

[1] Burchnall J.L., Chaundy T.W., Commutative ordinary differential operators, Proc. Lond. Math. Soc. s2-21 (1923), 420-440.

[2] Burchnall J.L., Chaundy T.W., Commutative ordinary differential operators, Proc. R. Soc. Lond. Ser. A 118 (1928), 557-583.

[3] Burchnall J.L., Chaundy T.W., Commutative ordinary differential operators. II. The identity $P^{n}=Q^{m}$, Proc. R. Soc. Lond. Ser. A 134 (1931), 471-485.

[4] Dixmier J., Sur les algèbres de Weyl, Bull. Soc. Math. France 96 (1968), 209-242.

[5] Dubrovin B.A., Periodic problems for the Korteweg-de Vries equation in the class of finite band potentials, Funct. Anal. Appl. 9 (1975), 215-223.

[6] Dubrovin B.A., Matveev V.B., Novikov S.P., Non-linear equations of Korteweg-de Vries type, finite-zone linear operators, and Abelian varieties, Russ. Math. Surv. 31 (1976), no. 1, 59-146.

[7] Grinevich P.G., Rational solutions for the equation of commutation of differential operators, Funct. Anal. Appl. 16 (1982), 15-19.

[8] Kasman A., Darboux transformations from n-KdV to KP, Acta Appl. Math. 49 (1997), $179-197$.

[9] Kasman A., Rothstein M., Bispectral Darboux transformations: the generalized Airy case, Phys. D 102 (1997), 159-176, q-alg/9606018.

[10] Krichever I.M., Commutative rings of ordinary linear differential operators, Funct. Anal. Appl. 12 (1978), 175-185.

[11] Krichever I.M., Integration of nonlinear equations by the methods of algebraic geometry, Funct. Anal. Appl. 11 (1977), 12-26.

[12] Krichever I.M., Methods of algebraic geometry in the theory of non-linear equations, Russ. Math. Surv. 32 (1977), no. 6, 185-213. 
[13] Krichever I.M., Novikov S.P., Holomorphic bundles and nonlinear equations. Finite-gap solutions of rank 2, Dokl. Akad. Nauk SSSR 247 (1979), 33-37.

[14] Krichever I.M., Novikov S.P., Holomorphic bundles over Riemann surfaces and the Kadomtsev-Petviashvili equation. I, Funct. Anal. Appl. 12 (1978), 276-286.

[15] Latham G.A., Previato E., Darboux transformations for higher-rank Kadomtsev-Petviashvili and KricheverNovikov equations, Acta Appl. Math. 39 (1995), 405-433.

[16] Latham G., Previato E., Higher rank Darboux transformations, in Singular Limits of Dispersive Waves (Lyon, 1991), NATO Adv. Sci. Inst. Ser. B Phys., Vol. 320, Plenum, New York, 1994, 117-134.

[17] Mironov A.E., A ring of commuting differential operators of rank 2 corresponding to a curve of genus $2, S b$. Math. 195 (2004), 711-722.

[18] Mironov A.E., Commuting rank 2 differential operators corresponding to a curve of genus 2, Funct. Anal. Appl. 39 (2005), 240-243.

[19] Mironov A.E., On commuting differential operators of rank 2, Sib. Èlektron. Mat. Izv. 6 (2009), 533-536.

[20] Mironov A.E., Self-adjoint commuting differential operators and commutative subalgebras of the Weyl algebra, arXiv:1107.3356.

[21] Mokhov O.I., Commuting differential operators of rank 3, and nonlinear equations, Math. USSR Izv. 35 (1990), 629-655.

[22] Mokhov O., On commutative subalgebras of the Weyl algebra that are related to commuting operators of arbitrary rank and genus, arXiv:1201.5979.

[23] Novikov S.P., The periodic problem for the Korteweg-de vries equation, Funct. Anal. Appl. 8 (1974), 236246.

[24] Novikov S.P., Grinevich P.G., Spectral theory of commuting operators of rank two with periodic coefficients, Funct. Anal. Appl. 16 (1982), 19-21.

[25] Previato E., Seventy years of spectral curves: 1923-1993, in Integrable Systems and Quantum Groups (Montecatini Terme, 1993), Lecture Notes in Math., Vol. 1620, Springer, Berlin, 1996, 419-481.

[26] Previato E., Wilson G., Differential operators and rank 2 bundles over elliptic curves, Compositio Math. 81 (1992), 107-119.

[27] Previato E., Wilson G., Vector bundles over curves and solutions of the KP equations, in Theta Functions Bowdoin 1987, Part 1 (Brunswick, ME, 1987), Proc. Sympos. Pure Math., Vol. 49, Amer. Math. Soc., Providence, RI, 1989, 553-569.

[28] Shabat A.B., Elkanova Z.S., Commuting differential operators, Theoret. and Math. Phys. 162 (2010), 276285.

[29] Wilson G., Bispectral commutative ordinary differential operators, J. Reine Angew. Math. 442 (1993), 177-204. 